\title{
Results of stereotactic radiosurgery of arteriovenous malformations: an analysis of 52 cases
}

\author{
ANDRAS A KEMENY, PALITHA S DIAS, DAVID M C FORSTER \\ From the Department of Neurosurgery, Royal Hallamshire Hospital, Sheffield, UK
}

SUMMARY The stereotactic radiosurgery unit in Sheffield became operational in September 1985 and over 180 patients harbouring AVMs have been treated. The first 52 patients underwent one year follow up angiography and comprise the material for this study. At one year 26 patients $(50 \%)$ already had a favourable outcome (16 complete and 10 almost complete obliteration). The results were the same in the two sexes. There was a better response in younger patients: under 20 years $75 \%$ favourable, between 20 and 40 years $45 \%$ favourable and above 40 years about $25 \%$ favourable. Malformations in a lateral position appeared to respond better than those in the midline. Lesions fed by vessels from more than one large vessel territory had less chance of complete obliteration at one year than those gaining blood from only one main supply. There was no difference in outcome between small malformations $\left(<2 \mathrm{~cm}^{3}\right)$ medium $\left(2-3 \mathrm{~cm}^{3}\right)$ or large $\left(>3 \mathrm{~cm}^{3}\right)$. No immediate morbidity or late side effects were encountered in these patients. Stereotactic radiosurgery is a safe and effective method in the treatment of arteriovenous malformations but there is a relatively long latency. The number of malformations obliterated is expected to be much higher when two years have elapsed after treatment.

Spontaneous thrombosis of arteriovenous malformations (AVMs) occurs only rarely. The incidence of thrombosis can be increased by conventional radiotherapy ${ }^{1-3}$ but the success rate is usually so low that it has been abandoned in most centres and used only occasionally as a last resort. ${ }^{45}$ The advent of stereotactic radiosurgery made it possible to direct higher doses of radiation more accurately to any intra-cranial target. $^{6}$ When radiosurgery was employed in the treatment of cerebral AVMs Steiner, using the Leksell system of intersecting gamma rays, showed a dramatic increase in the rate of thrombo-obliteration successfully achieved..$^{7-11}$

In September 1985 a third generation gamma unit of Leksell design (RBS 5000, Nucletec SA, Switzerland) became operational in Sheffield. This paper is an account of the results obtained by stereotactic radiosurgery in cerebral arteriovenous malformations one year after the treatment.

Address for reprint requests: David M C Forster, FRCS, Department of Neurosurgery, Royal Hallamshire Hospital, Glossop Rd, Sheffield S10 2JF, UK.

Received 19 September 1988.

Accepted 1 January 1989

\section{Clinical material}

Over 180 patients harbouring AVMs have been treated. The first 52 patients ( 27 females and 25 males) underwent angiography after one year and comprise the material for this study. (Subsequently it has been the practice to perform angiography only two years after treatment). The patients' ages at treatment ranged from 8 to 60 years (mean 29.7 years). The presenting event was an intracerebral, intraventricular and/or subarachnoid haemorrhage in 50 cases. Seventeen patients had had more than one bleed. Epilepsy was present in eight cases, this being the only symptom in two. Nine patients had undergone surgical removal of a haematoma and/or part of the AVM prior to the referral. None of these patients had received conventional or stereotactic radiosurgery previously.

\section{Radiological aspects}

All patients underwent diagnostic four vessel angiography at the referring centre or at our hospital before treatment. This study included no patients with multiple or dural AVMs or aneurysms of the vein of Galen. All malformations in this material were of "high flow" type. One young female had an accompanying aneurysm in the circle of Willis. Forty-four malformations were supratentorial and eight were in the posterior fossa. There were more lesions on the right than on the left side (26 vs 18) and eight lesions were in the midline. The midline lesions were located in the hypothalamus, corpus callosum, brainstem or in the cerebellar vermis. Twenty one 
lesions $(\mathbf{4 0} \%)$ had a vessel supply from more than one major arterial territory. Thirty lesions $(58 \%)$ received a contribution from the posterior cerebral artery, $20(38 \%)$ from the middle cerebral artery, $18(35 \%)$ from the anterior cerebral artery, $9(17 \%)$ from the internal carotid artery and $5(10 \%)$ from the basilar artery.

\section{Methods}

The procedure was begun in the radiology department. First a modified Leksell frame (Electa Instrument AB, Stockholm, Sweden) was applied to the outer table of the skull using four point fixation under local anaesthetic. General anaesthesia was only used in children aged under 12 years. With the frame in situ an angiogram was performed. If necessary, two of the main vessels were injected simultaneously to outline the lesion. The stereotactic target point was determined by superimposing standard isodose maps so that the $50 \%$ isodose curve corresponded to the periphery of the malformation. In AVMs of irregular shape two or more overlapping fields were used. Taking into account the position of the radiation field(s), the geometrical measurements of the skull and the activity of the cobalt sources the three-dimensional dose-distribution and the required treatment time were calculated on a Nimbus microcomputer. The dose prescribed to the periphery of the lesions was $2500 \mathrm{cGy}$.

The patients were then transferred to the treatment unit. The central body is a steel casting incorporating 201 separately collimated Cobalt-60 sources. Details of the equipment have been described elsewhere. ${ }^{12}$ When overlapping fields were needed the coordinates were reset before further radiation was given using the same or different collimator sizes. All treatments were completed in a single session before the stereotactic frame was removed. The patients were returned to the ward after treatment and discharged the following day. CT scans with contrast enhancement were obtained at 6 month intervals, and after one year follow up angiography was performed at the referring centre or at our unit. Having analysed the films the degree of obliteration was classified independently by a neurosurgical and a neuroradiological member of the team. The groups were defined as follows. "Complete" obliteration meant the return of an entirely normal intracranial circulation. "Almost complete" meant no visible arteriovenous fistula or early filling of veins but some abnormality such as the size of the feeding vessels was still apparent. The largest group, which was termed "Partial", included all those cases where there was considerable change in the size of the lesion but because of a persisting fistula failed to fulfil the criteria for the previous two categories. "Slight change" was defined as some slowing of the flow through the shunt or minimal reduction in size. The first two categories were very strict though the others were more subjective.

Complications. In the immediate postoperative period some patients with AVMs in the posterior fossa complained of nausea and vomiting but this never lasted more than 18 hours. During the one year of follow up none of these patients rebled. No patients who had not previously had fits developed epilepsy nor was any change in the nature or frequency of fits reported in those already suffering from seizures. No radionecrosis was observed on computed tomography. One patient developed hemiballismus on the contralateral side a few hours after the follow up angiography in the referring centre. CT scan showed a low density area in the basal ganglia. Her symptoms slowly resolved over a period of 3 to 6 months. It was felt that the complaint resulted from thrombo-emboli during angiography and was not a direct effect of radiation.

\section{Results}

The findings at the one year follow up angiography are summarised in fig 1 . Where the radio-obliteration was complete or almost complete it was considered a favourable result. Twenty six patients $(50 \%)$ had such a favourable outcome (16 complete and 10 almost complete). Only one patient did not show any response at all to treatment.

The distribution of the outcome was the same in the two sexes $\left(\chi^{2}=2 \cdot 135\right.$, d.f. $=4$, N.S.). The results were not influenced by previous surgery $\left(\chi^{2}=2 \cdot 17\right.$, d.f. $=4$ N.S. $)$, the presence of seizures $\left(\chi^{2}=3 \cdot 29\right.$, d.f. $=4$, N.S.) or whether the patient had had a haemorrhage $\left(\chi^{2}=3 \cdot 27\right.$, d.f. $=4$, N.S.). Age was an important factor (fig 2). There was a much better response under the age of $20(75 \%$ favourable) than between 20 and 40 (45\% favourable) and above 40 years (about $25 \%$ favourable.) $\left(\chi^{2}=15.7\right.$, d.f. $=8$, $\mathrm{p}<0.05$ ).

The size of the lesion ranged from the barely visible to more than $5.5 \mathrm{~cm}^{3}$ in volume. There was no difference in outcome between small $\left(<2 \mathrm{~cm}^{3}\right)$ medium $\left(2-3 \mathrm{~cm}^{3}\right)$ or large $\left(>3 \mathrm{~cm}^{3}\right)$ malformations $\left(\chi^{2}=5.04\right.$, d.f. $=8$, N.S., Fig 3$)$.

Thirty nine patients were treated with a single field but $13(25 \%)$ received a multi-field treatment. No connection between the number of fields and the results could be demonstrated.

The position of the AVM seemed to play a role. Malformations in a lateral position responded better than those in the midline $\left(\chi^{2}=9.73\right.$, d.f. $=4$, $\mathrm{p}<0.05$, fig 4 ). Lesions fed by vessels from more than one large vessel territory had less chance of complete

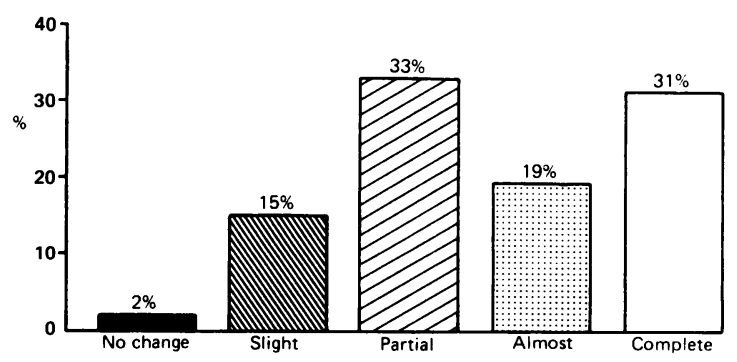

Fig 1 Results of stereotactic radiosurgery in arteriovenous malformations one year after treatment. "Complete" and "Almost complete" radio-obliteration are considered favourable results. 


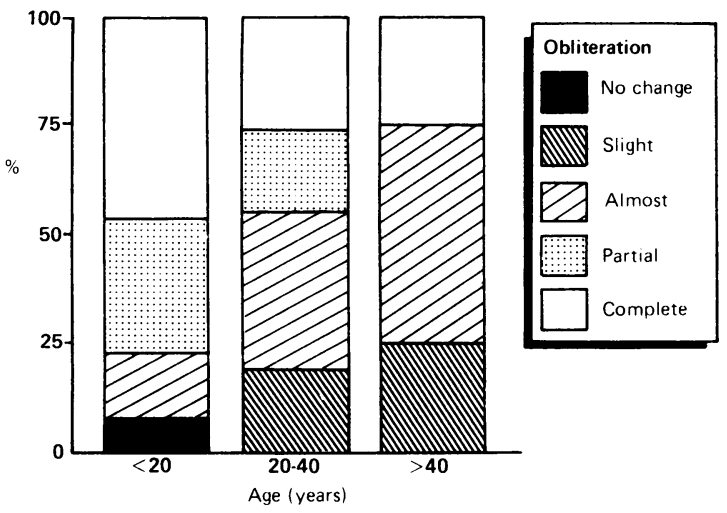

Fig 2 The rate of radio-obliteration one year after treatment in the different age groups. The height of each column represents the total of the patients $(100 \%)$ in each age group. The results were significantly better in younger patients $(p<0.05)$.

obliteration at one year than those gaining blood from only one main supplier $\left(\chi^{2}=10.63\right.$, d.f. $=4$, $\mathrm{p}<0.05)$.

\section{Discussion}

Cerebral arteriovenous malformations represent a threat to the patients' life and health from bleeding, epilepsy or progressive ischaemia. Published series of patients managed conservatively with a long follow up exceeding ten years show a mortality rate of 17$19 \%{ }^{1314}$ and the morbidity is much higher. Some patients suffer repeated haemorrhages and the psychological effect of this threat even if they survive unscathed should not be overlooked. Surgical excision of easily accessible malformations offers immediate

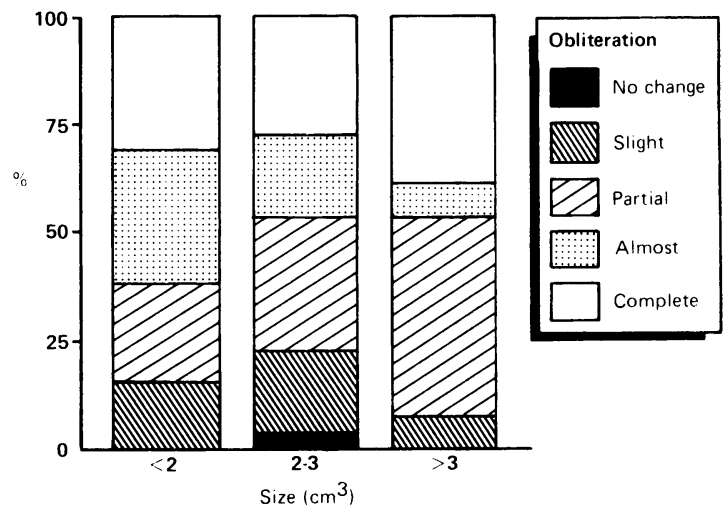

Fig 3 The rate of radio-obliteration in arteriovenous malformations of different sizes. The outcome was similar in small, medium and large lesions.

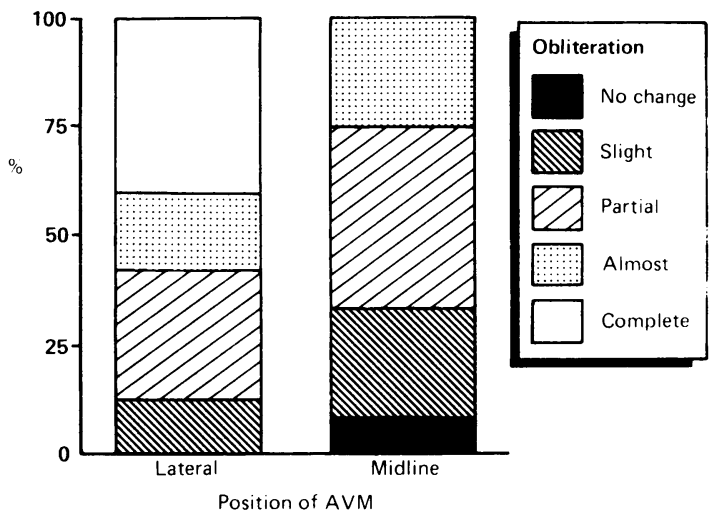

Fig 4 The effect of the position of the arteriovenous malformation on the results of radiosurgery after one year. The treatment was less successful in midline lesions (corpus callosum, brain stem, hypothalamus, cerebellar vermis) than in those situated in more lateral positions $(p<0.05)$.

and satisfactory protection but the open surgery of deep AVMs often presents considerable difficulties and dangers particularly in critical areas of the brain. ${ }^{15}$

Spontaneous thrombosis of AVMs is known to occur but is all too rare. The possibility of thrombosis can be increased by conventional radiotherapy, and has been tried over many years throughout the world ${ }^{1-3}$ Unfortunately, the results achieved were so disappointing that the method has been largely abandoned. However, when the Leksell radiosurgical system of delivering precisely high single doses of radiation $^{6}$ was applied to the treatment of AVMs significantly better results were obtained. ${ }^{7-11}$ Results have also been published with other systems of ionising radiation using synchrocyclotrons or linear accelerators $^{16-19}$ though not yet as good as those published using the Leksell system.

Based on the findings of Steiner in Stockholm ${ }^{10}$ the intention in this series was to expose the whole fistulous component of each AVM to a minimum dose of $2500 \mathrm{cGy}$, reduced only exceptionally in children under 10 years in whom, it is generally accepted, the tissues are more radiosensitive. Steiner had found that increasing the target dose to $7 \cdot 5-12.5 \mathrm{Krads}(7500$ $12500 \mathrm{cGy}$ ) increased the probability of radiation induced brain damage. ${ }^{10}$ All our patients received 5000 cGy at the target point.

In order to avoid radionecrosis of the brain, Steiner recommended stereotactic radiosurgery for arteriovenous malformations not larger than $3 \mathrm{~cm}^{3} .{ }^{10}$ This policy would leave many AVMs untreated. In Sheffield an $18 \mathrm{~mm}$ collimator system was available which gave a treated volume of $5 \mathrm{~cm}^{3}$ at the $50 \%$ isodose curve. Some lesions were treated which required more than one field. In this material there 
were 13 malformations larger than $3 \mathrm{~cm}^{3}$ and five of these were completely obliterated by one year. None of the 13 have had any side effects to date. It was also encouraging to find that the rate of thrombo-obliteration was equally good whether single or complex multi-field treatment plans were used.

Different regions of the brain have different radiosensitivities. In this study lesions in the midline appear to have responded less than those in a more lateral position. This finding does in fact correlate well with the observations made by Johnson ${ }^{2}$ following the use of conventional radiotherapy. Arteriovenous malformations in the midline are more likely to be supplied by vessels from more than one main arterial territory. Analysed in this way our material showed that malformations receiving blood from more than one main vessel were less likely to be obliterated than those supplied from one main vessel. It also is possible that not all the fistulous channels were completely visualised at the time of treatment despite comprehensive angiography and occasional simultaneous multivessel injection of contrast.

One year after treatment the chances of success appear to be greater in the younger age group. It will be of great interest to see if this difference persists after a longer follow up or whether it merely represents a shorter latency in youthful tissues.

The results obtained in Sheffield at one year were comparable to those in Stockholm. Already, a limited number of cases with angiography two years after treatment appear to follow the Swedish pattern $(82 \%$ complete obliteration) and full data will be published later.

The method does have some drawbacks and limitations. Lesions near the surface in patients with a large head may be difficult to position in the centre of the treatment field. There are some limitations in the size of AVM which can be irradiated this way although the maximum volume is clearly a changeable criterion. In addition shape and position as well as size have to be taken into account. The long latency before the onset of thrombo-obliteration means that there is a considerable period after treatment while the risk of a rebleed persists. On the other hand, the slow occlusion of the vessels and the associated delay may have the advantage of allowing collateral vessels to take over supply of the normal surrounding tissues and thus reduce the risk of neurological deficit from ischaemia. The incidence of late side effects in these patients has been remarkably low. Although no cases of radionecrosis were seen in this series one such patient has been encountered since. Steiner reported an incidence of 3-5\% among patients some of whom had received a higher dose of radiation. ${ }^{10}$ Clearly the risk is also higher in those patients who have already received some form of radiotherapy.
In conclusion, stereotactic radiosurgery is a safe and effective method for the treatment of cerebral arteriovenous malformations. The patients in this series have remained free from significant side effects. The procedure is simple, cost effective once the system has been established, hospitalisation is short and allows a fast turnover. In suitable circumstances the procedure could be carried out on an out-patient basis.

This work would not have been possible without the devoted assistance of the following: members of the Department of Radiotherapy (Drs JD Bradshaw and JJ Bolger, Therapy Radiotherapists Mrs S Seaman and Mrs P Hatland), Department of Neuroradiology (Drs U Bergvall and T Powell) and Medical Physics (L Walton, K Bomford and D Ramsden). Mrs J Fields provided invaluable secretarial help.

\section{References}

1 Johnson RT. Radiotherapy of cerebral angiomas, with a note on some problems in diagnosis. In Pia HW, Gleave JRW, Grote E et al, eds. Cerebral Angiomas. Advances in Diagnosis and Therapy. Heidelberg Springer Verlag, 1975:256-9.

2 Svien HJ, Peserico L. Regression in size of arteriovenous anomaly. $J$ Neurosurg 1960;17:493-6.

3 Tognetti F, Andreoli A, Cuscini A, Testa C. Successful management of an arteriovenous malformation by conventional irradiation. J Neurosurg 1985;63:193-5.

4 Cushing H, Bailey P. Tumors Arising from the BloodVessels of the Brain. Angiomatous Malformations and Haemangioblastomas. Springfield, Ill. Charles C Thomas, 1928:219.

5 Olivecrona H, Ladenheim J. Congenital Arteriovenous Aneurysms of the Carotid and Vertebral Arterial Systems. Berlin, Springer Verlag, 1957:50.

6 Leksell L. The stereotactic method and radiosurgery of the brain. Acta Neurochir Scand 1951;102:316-9.

7 Steiner L, Leksell T, Greitz D, Forster DMC, Backlund EO. Stereotactic radiosurgery for arteriovenous malformations. Acta Chir Scand 1972;138:459-64.

8 Steiner L, Leksell L, Forster DMC, Greitz T, Backlund EO. Stereotactic radiosurgery in intracranial arteriovenous malformations. Acta Neurochir 1974;Suppl 21:195-209.

9 Steiner L, Backlund EO, Greitz T, Leksell L, Noren G, Rahn T. Radiosurgery in intracranial arteriovenous malformations. II. A follow-up study. In: Carrea R, Le Vay D, eds. Neurological Surgery. Proceedings of the Sixth International Congress of Neurological Surgery, Sao Paulo, 1977. Amsterdam/Oxford: Excerpta Medica. 168-80.

10 Steiner L, Greitz T, Backlund EO, Leksell L, Noren G, Rahn T. Radiosurgery in arterio-venous malformations of the brain. Undue effects. In: Szikla G, ed. Stereotactic Cerebral Irradiation. (Inserm Symposium No 12, Amsterdam), Elsevier, 1979:257-40.

11 Steiner L. Radiosurgery in cerebral arteriovenous malfor- 
mations. In: Flamm E, Fein J, eds. Textbook of Cerebrovascular Surgery. New York, Springer Verlag NY Inc., vol 4, 1986:1161-215.

12 Walton L, Bomford CK, Ramsden D. The Sheffield stereotactic radiosurgery unit: physical characteristics and principles of operation. Br J Radiol 1987;60: 897-906.

13 Forster DMC, Steiner L, Hakanson S. Arteriovenous malformation of the brain. A long term clinical study. $J$ Neurosurg 1972;37:562-70.

14 Pool JL. Treatment of arteriovenous malformations of the cerebral hemispheres. $J$ Neurosurg 1962;19:136-41.

15 Kunc Z. Deep seated arteriovenous malformations: a critical review. In: Carrea R, ed. Neurological Surgery, with special emphasis on Non-Invasive Methods of Diagnosis and Treatment. Amsterdam: Excerpta
Medica 1978:12-23.

16 Larsson B, Leksell L, Rexed B, Sourander P, Mair W, Andersson B. The high energy proton beam as a neurosurgical tool. Nature 1958;182:1222-3.

17 Kjellberg RM. Stereotactic Bragg peak proton radiosurgery results. In: Szikla G, ed. Stereotactic Cerebral Irradiation. (Inserm Symposium No 12, Amsterdam), Elsevier, 1977:233-40.

18 Fabrikant JI, Lyman JT, Hosobuchi Y. Stereotactic heavy-ion Bragg peak radiosurgery for intra-cranial vascular disorders: method for treatment of deep arteriovenous malformations. $\mathrm{Br} J$ Radiol 1984; 57:479-90.

19 Colombo F, Benedetti A, Pozza F, et al. External stereotactic irradiation by linear accelerator. Neurosurgery 1985;16:154-60. 